\title{
Heat Flow from Polygons
}

\section{M. van den Berg ${ }^{1}$ P. B. Gilkey ${ }^{2}$ K. Gittins ${ }^{3}$}

Received: 15 February 2019 / Accepted: 6 August 2019 / Published online: 7 September 2019

(C) The Author(s) 2019

\section{Abstract}

We study the heat flow from an open, bounded set $D$ in $\mathbb{R}^{2}$ with a polygonal boundary $\partial D$. The initial condition is the indicator function of $D$. A Dirichlet 0 boundary condition has been imposed on some but not all of the edges of $\partial D$. We calculate the heat content of $D$ in $\mathbb{R}^{2}$ at $t$ up to an exponentially small remainder as $t \downarrow 0$.

Keywords Heat content · Polygon

Mathematics Subject Classification (2010) $35 \mathrm{~K} 05 \cdot 35 \mathrm{~K} 20$

\section{Introduction}

Let $D$ be an open, bounded set in $\mathbb{R}^{m}$ with finite Lebesgue measure $|D|$, and with boundary $\partial D$. We consider the heat equation

$$
\Delta u=\frac{\partial u}{\partial t},
$$

and impose a Dirichlet 0 boundary condition on $\partial D$. That is

$$
u(x ; t)=0, x \in \partial D, t>0 .
$$

We denote the (weak) solution corresponding to the initial datum

$$
\lim _{t \downarrow 0} u(x ; t)=1, x \in D,
$$

M. van den Berg

mamvdb@bristol.ac.uk

P. B. Gilkey

gilkey@uoregon.edu

K. Gittins

katie.gittins@unine.ch

1 School of Mathematics, University of Bristol, Fry Building, Woodland Road, Bristol BS8 1UG, UK

2 Mathematics Department, University of Oregon, Eugene, OR 97403, USA

3 Institut de Mathématiques, Université de Neuchâtel, Rue Emile-Argand 11, CH-2000

Neuchâtel, Switzerland 
by $u_{D}$. Then $u_{D}(x ; t)$ represents the temperature at $x \in D$ at time $t$ when $D$ has initial temperature 1 , and its boundary is kept at fixed temperature 0 . The heat content of $D$ at $t$ is denoted by

$$
Q_{D}(t)=\int_{D} d x u_{D}(x ; t) .
$$

Both $u_{D}$ and $Q_{D}(t)$ have been the subjects of a thorough investigation going back to the treatise by Carslaw and Jaeger, [9]. For a more recent account we refer to [2, 13].

Many different versions and extensions have already been considered. For example, the case where $\partial D$ is smooth, and $A$ is an open subset of $\partial D$ on which a Neumann (insulating) boundary condition has been imposed, while the temperature 0 Dirichlet condition has been maintained on $\partial D-A$. This Zaremba boundary condition for the heat equation has been considered in [4], for example. Even in the case where no boundary condition has been imposed on $\partial D$, the corresponding heat content, denoted by $H_{D}(t)$, has (if $\partial D$ is smooth) an asymptotic series as $t \downarrow 0$ similar to the one for $Q_{D}(t)$, see [3], for example.

In this paper we consider the heat flow out of $D$ into $\mathbb{R}^{m}$, where a Dirichlet 0 boundary condition has been imposed on a closed subset $\partial D_{-} \subset \partial D$, and where no boundary condition has been imposed on $\partial D_{+}:=\partial D-\partial D_{-}$. That is

$$
\Delta u=\frac{\partial u}{\partial t},
$$

with boundary condition

$$
u(x ; t)=0, x \in \partial D_{-}, t>0 .
$$

We denote the solution corresponding to the initial datum

$$
\lim _{t \downarrow 0} u(x ; t)=\mathbf{1}_{D}(x) \text {, almost everywhere, }
$$

by $u_{D, \partial D_{-}}$. Here $\mathbf{1}_{D}$ is the indicator function of $D$. Then $u_{D, \partial D_{-}}$is the weak solution of Eqs. 1.1, 1.2 and 1.3, where Eq. 1.2 holds at all regular points of $\partial D_{-}$. The open set $D$ looses heat via two mechanisms: (i) part of the boundary, $\partial D_{-}$, is at fixed temperature 0 , and cools the interior of $D$; (ii) since the complement of $D$ is at initial temperature 0 , heat flows over the open part of the boundary, $\partial D_{+}$. The corresponding heat content is denoted by

$$
G_{D, \partial D_{-}}(t)=\int_{D} d x u_{D, \partial D_{-}}(x ; t) .
$$

Let $A$ be a closed subset of $\mathbb{R}^{m}$, and let $p_{\mathbb{R}^{m}-A}(x, y ; t), x \in \mathbb{R}^{m}-A, y \in \mathbb{R}^{m}-A, t>0$ be the heat kernel for the open set $\mathbb{R}^{m}-A$ with a Dirichlet 0 boundary condition on $A$. This heat kernel is non-negative, symmetric in its space variables, and satisfies the heat semigroup property. Moreover, If $A$ and $B$ are closed subsets with $B \subset A$ then $p_{\mathbb{R}^{m}-A}(x, y ; t) \leq$ $p_{\mathbb{R}^{m}-B}(x, y ; t), x \in \mathbb{R}^{m}-A, y \in \mathbb{R}^{m}-A, t>0$. We refer to [12] for further details. Then for $x \in D$

$$
u_{D, \partial D_{-}}(x ; t)=\int_{D} d y p_{\mathbb{R}^{m}-\partial D_{-}}(x, y ; t) .
$$

Let $\left(B(s), s \geq 0, \mathbb{P}_{x}, x \in \mathbb{R}^{m}\right)$ be Brownian motion associated with $\Delta$. Recall that $p_{\mathbb{R}^{m}-\partial D_{-}}$is the transition density for Brownian motion on $\mathbb{R}^{m}$ with killing on $\partial D_{-}$. If $\tau_{\partial D_{-}}=\left\{\inf s \geq 0: B(s) \in \partial D_{-}\right\}$, then

$$
u_{D, \partial D_{-}}(x ; t)=\mathbb{P}_{x}\left(\tau_{\partial D_{-}} \geq t, B(t) \in D\right),
$$

which jibes with Eq. 1.4.

Since $D \subset \mathbb{R}^{m}-\partial D_{-} \subset \mathbb{R}^{m}$, we have by monotonicity,

$$
0 \leq u_{D}(x ; t)=u_{D, \partial D}(x ; t) \leq u_{D, \partial D_{-}}(x ; t) \leq u_{D, \emptyset}(x ; t) .
$$


Hence

$$
Q_{D}(t) \leq G_{D, \partial D_{-}}(t) \leq H_{D}(t), t>0 .
$$

Using the spectral resolution for the Dirichlet heat kernel on $\mathbb{R}^{m}-\partial D_{-}$it is possible to show that all three heat contents in Eq. 1.6 are strictly decreasing in $t$. Moreover, Eq. 1.3 implies that for $1 \leq p<\infty$,

$$
\lim _{t \downarrow 0}\left\|u_{D, \partial D_{-}}(\cdot ; t)-\mathbf{1}_{D}(\cdot)\right\|_{L^{p}\left(\mathbb{R}^{m}-\partial D_{-}\right)}=0 .
$$

The short proof below is instructive. See also [5]. By monotonicity,

$$
p_{\mathbb{R}^{m}-\partial D_{-}}(x, y ; t) \leq p_{\mathbb{R}^{m}}(x, y ; t)=(4 \pi t)^{-m / 2} e^{-|x-y|^{2} /(4 t)} .
$$

Hence $0<u_{D, \partial D_{-}}(x ; t) \leq 1$, and $\left|u_{D, \partial D_{-}}(x ; t)-1\right| \leq 1$. Moreover,

$$
\begin{aligned}
\left\|u_{D, \partial D_{-}}(\cdot ; t)-\mathbf{1}_{D}(\cdot)\right\|_{L^{p}\left(\mathbb{R}^{m}\right)}^{p}= & \int_{D} d x\left|u_{D, \partial D_{-}}(x ; t)-1\right|^{p}+\int_{\mathbb{R}^{m}-D} d x u_{D, \partial D_{-}}(x ; t)^{p} \\
\leq & \int_{D} d x\left|u_{D, \partial D_{-}}(x ; t)-1\right|+\int_{\mathbb{R}^{m}-D} d x u_{D, \partial D_{-}}(x ; t) \\
= & \int_{D} d x\left|u_{D, \partial D_{-}}(x ; t)-1\right|+\int_{\mathbb{R}^{m}} d x u_{D, \partial D_{-}}(x ; t) \\
& -\int_{D} d x u_{D, \partial D_{-}}(x ; t) .
\end{aligned}
$$

By Eq. 1.4, Tonelli's theorem, and monotonicity,

$$
\begin{aligned}
\int_{\mathbb{R}^{m}} d x u_{D, \partial D_{-}}(x ; t) & =\int_{D} d y \int_{\mathbb{R}^{m}} d x p_{\mathbb{R}^{m}-\partial D_{-}}(x, y ; t) \\
& \leq \int_{D} d y \int_{\mathbb{R}^{m}} d x p_{\mathbb{R}^{m}}(x, y ; t)=\int_{D} d y .
\end{aligned}
$$

By Eqs. 1.9 and 1.10,

$$
\left\|u_{D, \partial D_{-}}(\cdot ; t)-\mathbf{1}_{D}(\cdot)\right\|_{L^{p}\left(\mathbb{R}^{m}\right)}^{p} \leq 2 \int_{D} d x\left|1-u_{D, \partial D_{-}}(x ; t)\right|,
$$

and Eq. 1.7 follows by Lebesgue's Dominated Convergence theorem and Eq. 1.3.

The main results of this paper are concerned with the special case where $D$ is an open, bounded set in $\mathbb{R}^{2}$ with a polygonal boundary. Throughout we make the hypothesis that the vertices of $\partial D$ are the endpoints of exactly two edges, and that the collection of vertices $\mathcal{V}=\left\{V_{1}, V_{2}, \cdots\right\}$ is finite. We consider edges of two types: Dirichlet edges which include their endpoints, and open edges which include those vertices common to two open edges. The union of all Dirichlet edges, denoted by $\partial D_{-}$as above, is a closed subset of $\mathbb{R}^{2}$, and we denote its length by $L\left(\partial D_{-}\right)$. The union of all open edges, denoted by $\partial D_{+}$, is a relatively open subset of $\partial D$. We denote its length by $L\left(\partial D_{+}\right)$. The length of $\partial D$ is given by

$$
L(\partial D)=L\left(\partial D_{-}\right)+L\left(\partial D_{+}\right) .
$$

It was shown in [8] that if all edges are of Dirichlet type, then

$$
Q_{D}(t)=|D|-\frac{2}{\pi^{1 / 2}} L(\partial D) t^{1 / 2}+\sum_{\gamma \in \mathcal{C}} c(\gamma) t+O\left(e^{-q_{D} / t}\right), t \downarrow 0,
$$

where $q_{D}>0$ is a constant which depends on $D$ only, $c:(0,2 \pi] \rightarrow \mathbb{R}$ is defined by

$$
c(\gamma)=\int_{0}^{\infty} d \theta \frac{4 \sinh ((\pi-\gamma) \theta)}{\sinh (\pi \theta) \cosh (\gamma \theta)}
$$


$\mathcal{C}=\left\{\gamma_{1}, \gamma_{2}, \ldots\right\}$ are the interior angles at the vertices $V_{1}, V_{2}, \ldots$, and $L(\partial D)$ is the total length of all Dirichlet edges.

On the other hand, if all edges are of open type, that is $\partial D_{-}=\emptyset$, then it was shown in [6] that

$$
H_{D}(t)=|D|-\frac{1}{\pi^{1 / 2}} L(\partial D) t^{1 / 2}+\sum_{\beta \in \mathcal{B}} b(\beta) t+O\left(e^{-h_{D} / t}\right), t \downarrow 0,
$$

where $h_{D}>0$ is a constant which depends on $D$ only, $b:(0,2 \pi) \rightarrow \mathbb{R}$ is defined by

$$
b(\beta)= \begin{cases}\frac{1}{\pi}+\left(1-\frac{\beta}{\pi}\right) \cot \beta, & \beta \in(0, \pi) \cup(\pi, 2 \pi) ; \\ 0, & \beta=\pi,\end{cases}
$$

$\mathcal{B}=\left\{\beta_{1}, \beta_{2}, \ldots\right\}$ are the interior angles at the vertices $V_{1}, V_{2}, \ldots$, and $L(\partial D)$ is the total length of all open edges.

The main result of this paper, Theorem 1.1 below, allows both open and Dirichlet edges. The collection of interior angles between two adjacent Dirichlet, respectively open, edges is denoted by $\mathcal{C}$, respectively $\mathcal{B}$. The collection of angles between an adjacent pair of openDirichlet edges (or Dirichlet-open edges) is denoted by $\mathcal{A}$ (see Fig. 1).

Theorem 1.1 There exists a constant $g_{D}>0$ depending on $D$ only such that

$$
\begin{aligned}
G_{D, \partial D_{-}}(t)= & |D|-\frac{1}{\pi^{1 / 2}}\left(2 L\left(\partial D_{-}\right)+L\left(\partial D_{+}\right)\right) t^{1 / 2} \\
& +\left(\sum_{\gamma \in \mathcal{C}} c(\gamma)+\sum_{\beta \in \mathcal{B}} b(\beta)+\sum_{\alpha \in \mathcal{A}} a(\alpha)\right) t+O\left(e^{-g_{D} / t}\right), t \downarrow 0,
\end{aligned}
$$

where $a:(0,2 \pi) \mapsto \mathbb{R}$ is given by

$$
a(\alpha)=-\frac{3}{4}+\frac{1}{4} \int_{0}^{\infty} d \theta \frac{4\left(\sinh \left(\left(\pi-\frac{\alpha}{2}\right) \theta\right)\right)^{2}-(\sinh ((\pi-\alpha) \theta))^{2}}{(\sinh (\pi \theta / 2))^{2} \cosh (\pi \theta)} .
$$

The main results of both [6] and [8] hold for more general polygons. For example, vertices with just one edge or more than two are allowed. If a vertex supports just one edge,

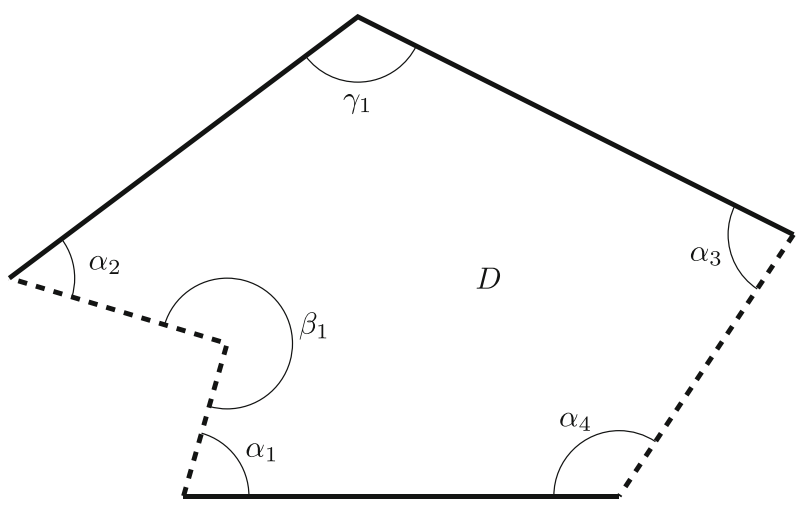

Fig. 1 An open set $D \subset \mathbb{R}^{2}$ with polygonal boundary: the Dirichlet, respectively open, edges are displayed as solid, respectively dashed, lines 
then the corresponding angle equals $2 \pi$ and will contribute $c(2 \pi)$ to the coefficient of $t$ in Eq. 1.12. That edge counts double in the total length of Dirichlet edges. Indeed, that edge cools $D$ at both sides. In general, the contribution from the angles to the coefficient $t$ in Eq. 1.12 is additive. The Dirichlet condition on the edges implies this additivity. That does not hold true in the setting of open edges. If two wedges with angles, say $\beta_{1}$ and $\beta_{2}$, are supported by the same vertex, then there is an additional contribution to the coefficient of $t$, depending on $\beta_{1}, \beta_{2}$ and the angle between these two wedges (see [6]). Furthermore, if a vertex supports just one edge, then the corresponding angle, and the corresponding edge contribute 0 to the coefficients of $t$ and $t^{1 / 2}$ respectively. Indeed, heat does not flow over this edge into $\mathbb{R}^{2}-D$. We shall not consider these cases, and we assume that each vertex supports precisely two edges.

The proof of Theorem 1.1 is based on a partition of $D$ combined with model computations, as are the proofs of Eqs. 1.12 and 1.14. The main computation is the one for circular sectors with radius $R$ with opening angles $\gamma, \beta, \alpha$ depending on whether one deals with a Dirichlet-Dirichlet wedge, an open-open wedge, or, as in this paper, a Dirichlet-open wedge. The geometry of the Dirichlet-open wedge is one edge on which a Dirichlet boundary condition has been imposed, and an open edge separated by angle $\alpha$ (see Fig. 2). Our main result for such a circular sector is the following.

Theorem 1.2 Let $W_{\alpha}=\{(r, \phi): r>0,0<\phi<\alpha\}$ in polar coordinates, and let $u_{W_{\alpha}}((r, \phi) ; t)$ be the solution of the heat equation with a Dirichlet 0 boundary condition on the positive $x_{1}$ axis, and initial data $\boldsymbol{1}_{W_{\alpha}}$. Then, in polar coordinates, we have

$$
\begin{aligned}
\int_{0}^{R} d r r \int_{0}^{\alpha} d \phi u_{W_{\alpha}}((r, \phi) ; t)= & \frac{1}{2} \alpha R^{2}-\frac{3}{\pi^{1 / 2}} R t^{1 / 2}+a(\alpha) t \\
& +\frac{3 R t^{1 / 2}}{\pi^{1 / 2}} \int_{1}^{\infty} \frac{d v}{v^{2}} \int_{0}^{1} d \zeta \frac{\zeta}{\left(1-\zeta^{2}\right)^{1 / 2}} e^{-R^{2} \zeta^{2} v^{2} /(4 t)} \\
& +O\left(e^{-m_{\alpha} R^{2} /(4 t)}\right), t \downarrow 0
\end{aligned}
$$

where $m_{\alpha}>0$ is a constant which depends on $\alpha$ only.

We recognise the various terms in the right-hand side as follows. The first term is the area of the circular sector with opening angle $\alpha$ and radius $R$. The second term combines the contributions from an open edge of length $R$, and a Dirichlet edge of length $R$. The latter having an extra factor 2 . The third term is the angle contribution. The fourth term represents the contribution from two cusps. See Section 2 for details.

Fig. 2 A Dirichlet-open wedge with angle $\alpha$ : the Dirichlet, respectively open, edge is displayed as a solid, respectively dashed, line

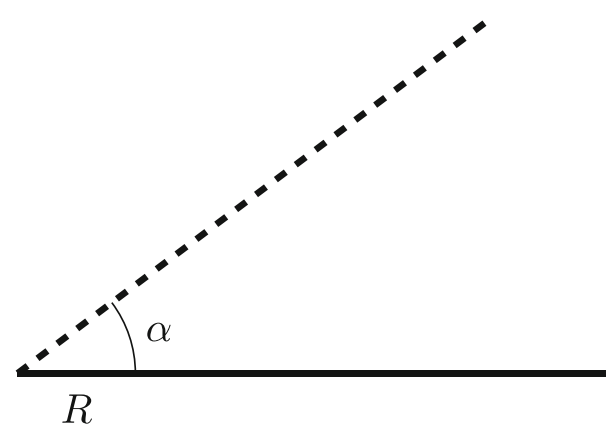


Unlike the integral for $c(\gamma)$ in Eq. 1.13, it is possible to evaluate the expression for $a(\alpha)$ in Eq. 1.16. To do so we write

$$
\frac{1}{(\sinh (\pi \theta / 2))^{2} \cosh (\pi \theta)}=\frac{1}{(\sinh (\pi \theta / 2))^{2}}-\frac{2}{\cosh (\pi \theta)},
$$

and compute the resulting four integrals using formulae 3.511.7 and 3.511.9 in [14]. The common range of convergence for these four integrals is $\pi<\alpha<3 \pi / 2$. We find

$a(\alpha)=-\frac{3}{8}+\frac{3}{4 \pi}-\frac{1}{8 \cos \alpha}+\frac{1}{2 \cos (\alpha / 2)}+\left(\frac{7}{4}-\frac{3 \alpha}{4 \pi}\right) \frac{1}{\tan \alpha}+\left(\frac{1}{4}-\frac{\alpha}{4 \pi}\right) \tan \alpha, \pi<\alpha<3 \pi / 2$.

Outside this interval we can use Eq. 1.16 to evaluate $a(\alpha)$. For example, we have

$$
\begin{gathered}
a(\pi / 2)=-\frac{3}{8}+\frac{1}{\pi}+\frac{1}{2} \sqrt{2}, \\
a(\pi)=-\frac{1}{4}, \\
a(3 \pi / 2)=-\frac{3}{8}+\frac{1}{\pi}-\frac{1}{2} \sqrt{2} .
\end{gathered}
$$

The value $a(\pi)=-\frac{1}{4}$ in Eq. 1.18 is of particular interest. Consider an open, bounded set $D$ in $\mathbb{R}^{m}$ with $C^{\infty}$ boundary $\partial D$. Let $\partial D_{-}$be a closed subset of $\partial D$ with $C^{\infty}$ boundary $\Sigma$, and $\operatorname{dim} \Sigma=m-2$. Let $u_{D, \partial D_{-}}$be the solution of Eqs. 1.1, 1.2, and 1.3. Then, provided an asymptotic series in half powers of $t$ exists, we have

$$
\begin{aligned}
G_{D, \partial D_{-}}(t)= & |D|-\pi^{-1 / 2}\left(2 \int_{\partial D_{-}} d \sigma+\int_{\partial D-\partial D_{-}} d \sigma\right) t^{1 / 2} \\
& +\left(\frac{1}{2} \int_{\partial D_{-}} d \sigma L_{a a}(\sigma)-\frac{1}{4} \operatorname{vol}(\Sigma)\right) t+O\left(t^{3 / 2}\right), t \downarrow 0,
\end{aligned}
$$

where $d \sigma$ denotes the surface measure on $\partial D, L_{a a}$ is the trace of the second fundamental form defined by the inward unit normal vector field of $\partial D$ in $D, \operatorname{vol}(\Sigma)$ is the $(m-2)$ dimensional volume of the boundary of $\partial D_{-}$in $\partial D$, and $a(\pi)$ is its coefficient. To see that Eq. 1.19 holds, we note that the local geometry around $\Sigma$ is as follows. Let $P$ be a point of $\Sigma$. Then straightening out the boundary of $\partial D$ around $P$ we obtain, locally, an $(m-1)$ dimensional hyper plane. The straightening out of $\Sigma$ around $P$ partitions this hyper plane into two hyper half-planes at angle $\pi$. On one (closed) hyper half-plane we have a Dirichlet 0 boundary condition, and on the remaining open hyper half-plane we do not have boundary conditions. This is precisely the geometry of a Dirichlet-open wedge with angle $\pi$ times $\Sigma$. This then leads to the $a(\pi) \operatorname{vol}(\Sigma) t$ contribution in Eq. 1.19. The computation of the coefficient of $t^{3 / 2}$ promises to be more complicated even in this special setting. One expects that there is an integral over $\Sigma$ involving both the second fundamental form of $\Sigma$ in $D$ and the second fundamental form of $\partial D$ in $D$. Consequently, several special case calculations would be required. See also [4].

The proofs of Theorems 1.1 and 1.2 have been deferred to Sections 2, and 4 respectively. In Section 3 we state some technical preliminaries which will be used in the proof of Theorem 1.2.

\section{Proof of Theorem 1.1}

In this section, we make use of Theorem 1.2. We prove that the latter theorem holds in Section 4. 
Kac's principle of not feeling the boundary asserts that the solution of the heat equation with initial datum $\mathbf{1}_{D}$, where $D$ is an open set in $\mathbb{R}^{m}$, is equal to 1 on the interior of $D$ up to an exponentially small remainder, as $t \downarrow 0$. Kac formulated his principle in the case where a Dirichlet 0 boundary condition is imposed on all of $\partial D$, that is $\partial D_{+}=\emptyset$. It has been shown that it also holds if no boundary condition is imposed on $\partial D$, that is $\partial D_{-}=\emptyset$. See, for example, Proposition 9(i) in [1]. In the same spirit, we have the following lemma.

Lemma 2.1 If $D$ is an open set in $\mathbb{R}^{2}$, and if $\partial D_{-}$is a closed subset of $\partial D$, then

$$
1 \geq \int_{D} d y p_{\mathbb{R}^{2}-\partial D_{-}}(x, y ; t) \geq \int_{D} d y p_{D}(x, y ; t) \geq 1-2 e^{-d(x, \partial D)^{2} /(4 t)} .
$$

Proof Since the Dirichlet heat kernel is monotone in the domain, and since $D \subset \mathbb{R}^{m}-\partial D_{-}$,

$$
p_{\mathbb{R}^{m}-\partial D_{-}}(x, y ; t) \geq p_{D}(x, y ; t)>0, x \in D, y \in D, t>0 .
$$

Hence $u_{D, \partial D_{-}}(x ; t) \geq \int_{D} d y p_{D}(x, y ; t)$. The latter integral has been bounded from below in Lemma 4 of [8]. Taking $m=2$ in the first line of (3.2) in that paper we find Eq. 2.1. The upper bound in Eq. 2.1 follows as $p_{\mathbb{R}^{m}-\partial D_{-}}(x, y ; t) \leq p_{\mathbb{R}^{m}}(x, y ; t)$ and $\int_{\mathbb{R}^{m}} d y p_{\mathbb{R}^{m}}(x, y ; t)=1$.

As in [6-8], the strategy of the proof of Theorem 1.1 is to partition $D$ into sets on which $u_{D, \partial D_{-}}(x ; t)$ is approximated either by 1 , or by $u_{W_{\alpha}}(x ; t)$, or by $u_{H}(x ; t)$ (where $H \subset \mathbb{R}^{m}$ is a half-space) depending on where $x \in D$ lies with respect to the partition. By Lemma 2.1, the terms which compensate for these approximations are exponentially small.

Below we describe the partition of the set $D$. At each vertex of $\partial D$ with angle $\theta$, we consider the circular sector of radius $R>0$ and angle $\theta$ that is contained in $D$. For $\delta>0$ (to be specified later), we consider the set of points in $D$ that are at distance less than $\delta$ from $\partial D$ and that are not contained in the union of the circular sectors (see Fig. 3).

Let $H=\left\{\left(x_{1}, x_{2}\right) \in \mathbb{R}^{2}: x_{2}>0\right\}$. Up to changing the coordinates (if necessary), we can suppose that $\partial D \cap \partial H$ is an edge $e$ of length $\ell$. Let $L=\ell-2 R$. In this way, each blue region in Fig. 3 can be written (up to a set of measure 0 ) as the union of a rectangle

$$
\left\{\left(x_{1}, x_{2}\right) \in \mathbb{R}^{2}: R<x_{1}<R+L, 0<x_{2}<\delta\right\},
$$

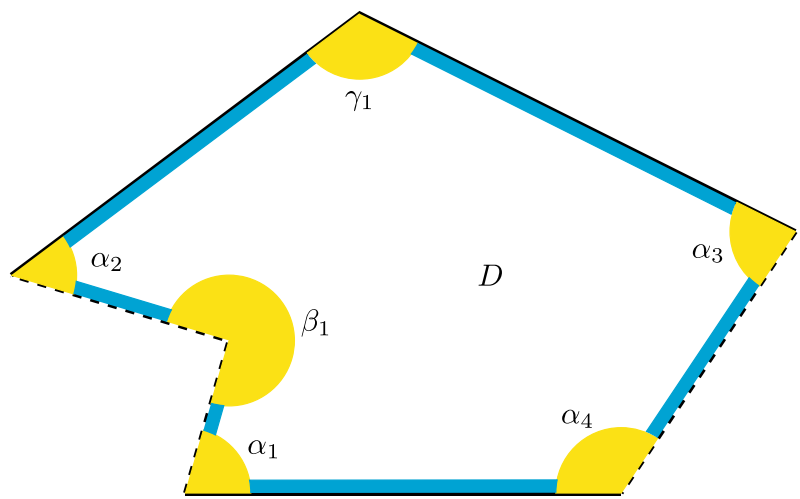

Fig. 3 Partition of $D$ (the Dirichlet, respectively open, edges are displayed as solid, respectively dashed, lines) 
and two cusps of the form

$$
E_{1}(\delta, R)=\left\{x \in \mathbb{R}^{2}: 0<x_{1}<R,|x|>R, 0<x_{2}<\delta\right\},
$$

and

$$
E_{2}(\delta, R)=\left\{x \in \mathbb{R}^{2}: \ell-R<x_{1}<\ell,|x-(\ell, 0)|>R, 0<x_{2}<\delta\right\} .
$$

We say that these cusps are adjacent to $\partial H$.

We observe that each sector has two neighbouring cusps. In the partition of $D$, cusps of two types feature. That is, those cusps adjacent to $\partial H$ with a Dirichlet 0 boundary condition on $e$, and those cusps adjacent to $\partial H$ without a boundary condition on $e$ (see Fig. 4). Cusps of the latter type feature in [6], and those of the former type feature in [8].

We first consider the case of a cusp which is adjacent to $\partial H$ with a Dirichlet 0 boundary condition.

\section{Lemma 2.2 If $\delta<R$ then}

$$
\begin{aligned}
\int_{E(\delta, R)} d x u_{H}(x ; t)= & |E(\delta, R)|-\frac{2 R t^{1 / 2}}{\pi^{1 / 2}} \int_{1}^{\infty} \frac{d w}{w^{2}} \int_{0}^{1} \frac{v d v}{\left(1-v^{2}\right)^{1 / 2}} e^{-R^{2} v^{2} w^{2} /(4 t)} \\
& +O\left(t^{1 / 2} e^{-\delta^{2} /(4 t)}\right) .
\end{aligned}
$$

Proof See also (4.7) in [8]. We have that

$$
u_{H}(x ; t)=\frac{1}{(\pi t)^{1 / 2}} \int_{0}^{x_{2}} d q e^{-q^{2} /(4 t)} .
$$

Since the length of the line segment in $E(\delta, R)$ parallel to the $x_{1}$ axis equals $R-\left(R^{2}-x_{2}^{2}\right)^{1 / 2}$, we have

$$
\begin{aligned}
\int_{E(\delta, R)} d x u_{H}(x ; t) & =\frac{1}{(\pi t)^{1 / 2}} \int_{0}^{\delta} d x_{2}\left(R-\left(R^{2}-x_{2}^{2}\right)^{1 / 2}\right) \int_{0}^{x_{2}} d q e^{-q^{2} /(4 t)} \\
& =|E(\delta, R)|-\frac{1}{(\pi t)^{1 / 2}} \int_{0}^{\delta} d x_{2}\left(R-\left(R^{2}-x_{2}^{2}\right)^{1 / 2}\right) \int_{x_{2}}^{\infty} d q e^{-q^{2} /(4 t)}
\end{aligned}
$$

Fig. 4 A sector contained in a Dirichlet-open wedge with angle $\alpha$, and its neighbouring cusps $E_{R}(\delta, R)$ adjacent to an edge with a Dirichlet 0 boundary condition, and $E_{B}(\delta, R)$ adjacent to an open edge (the Dirichlet, respectively open, edge is displayed as a solid, respectively dashed, line)

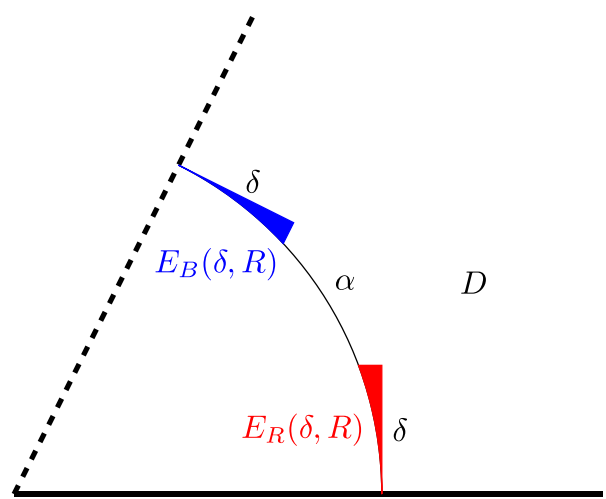




$$
\begin{aligned}
= & |E(\delta, R)|-\frac{1}{(\pi t)^{1 / 2}} \int_{0}^{\delta} d x_{2}\left(R-\left(R^{2}-x_{2}^{2}\right)^{1 / 2}\right) x_{2} \int_{1}^{\infty} d w e^{-w^{2} x_{2}^{2} /(4 t)} \\
= & |E(\delta, R)|-\frac{2 t^{1 / 2}}{\pi^{1 / 2}} \int_{1}^{\infty} \frac{d w}{w^{2}} \int_{0}^{\delta} \frac{x_{2} d x_{2}}{\left(R^{2}-x_{2}^{2}\right)^{1 / 2}} e^{-w^{2} x_{2}^{2} /(4 t)} \\
& +\frac{2 t^{1 / 2}}{\pi^{1 / 2}}\left(R-\left(R^{2}-\delta^{2}\right)^{1 / 2}\right) \int_{1}^{\infty} \frac{d w}{w^{2}} e^{-w^{2} \delta^{2} /(4 t)} \\
= & |E(\delta, R)|-\frac{2 R t^{1 / 2}}{\pi^{1 / 2}} \int_{1}^{\infty} \frac{d w}{w^{2}} \int_{0}^{1} \frac{v d v}{\left(1-v^{2}\right)^{1 / 2}} e^{-R^{2} v^{2} w^{2} /(4 t)} \\
& +\frac{2 t^{1 / 2}}{\pi^{1 / 2}}\left(R-\left(R^{2}-\delta^{2}\right)^{1 / 2}\right) \int_{1}^{\infty} \frac{d w}{w^{2}} e^{-w^{2} \delta^{2} /(4 t)} \\
& +\frac{2 t^{1 / 2}}{\pi^{1 / 2}} \int_{1}^{\infty} \frac{d w}{w^{2}} \int_{\delta}^{R} \frac{x_{2} d x_{2}}{\left(R^{2}-x_{2}^{2}\right)^{1 / 2}} e^{-w^{2} x_{2}^{2} /(4 t)} .
\end{aligned}
$$

Both the third and fourth terms in the right-hand side of Eq. 2.2 are $O\left(t^{1 / 2} e^{-\delta^{2} /(4 t)}\right)$.

Next we consider the case of a cusp which is adjacent to $\partial H$ which is open (that is without a boundary condition).

Lemma 2.3 If $\delta<R$, then

$$
\begin{aligned}
\int_{E(\delta, R)} d x u_{H, \emptyset}(x ; t)= & |E(\delta, R)|-\frac{R t^{1 / 2}}{\pi^{1 / 2}} \int_{1}^{\infty} \frac{d w}{w^{2}} \int_{0}^{1} \frac{v d v}{\left(1-v^{2}\right)^{1 / 2}} e^{-R^{2} v^{2} w^{2} /(4 t)} \\
& +O\left(t^{1 / 2} e^{-\delta^{2} /(4 t)}\right)
\end{aligned}
$$

Proof We recall that for $D \subset \mathbb{R}^{m}$ open,

$$
u_{D, \emptyset}(x ; t)=\int_{D} d y(4 \pi t)^{-m / 2} e^{-|x-y|^{2} /(4 t)},
$$

(see [1] for example). Hence, for $D=H$, we have

$$
u_{H, \emptyset}(x ; t)=1-\frac{1}{(4 \pi t)^{1 / 2}} \int_{x_{2}}^{\infty} d q e^{-q^{2} /(4 t)} .
$$

Comparing Eq. 2.4 with

$$
u_{H}(x ; t)=1-\frac{1}{(\pi t)^{1 / 2}} \int_{x_{2}}^{\infty} d q e^{-q^{2} /(4 t)},
$$

we see that the second, third and fourth terms in the right-hand side of Eq. 2.2 are weighted with a factor $\frac{1}{2}$ in the computation of the integral in the left-hand side of Eq. 2.3. This then gives Eq. 2.3.

Lemma 2.4 If $S=\left\{\left(x_{1}, x_{2}\right) \in \mathbb{R}^{2}: 0<x_{1}<L, 0<x_{2}<\delta\right\}$, then

$$
\int_{S} d x u_{H}(x ; t)=|S|-\frac{2 L t^{1 / 2}}{\pi^{1 / 2}}+O\left(t^{1 / 2} e^{-\delta^{2} /(4 t)}\right),
$$


and

$$
\int_{S} d x u_{H, \emptyset}(x ; t)=|S|-\frac{L t^{1 / 2}}{\pi^{1 / 2}}+O\left(t^{1 / 2} e^{-\delta^{2} /(4 t)}\right) .
$$

Proof We have

$$
\begin{aligned}
\int_{S} d x u_{H}(x ; t) & =\int_{0}^{L} d x_{1} \int_{0}^{\delta} d x_{2}\left(1-\frac{1}{(\pi t)^{1 / 2}} \int_{x_{2}}^{\infty} d q e^{-q^{2} /(4 t)}\right) \\
& =|S|-\frac{2 L t^{1 / 2}}{\pi^{1 / 2}}+\int_{\delta}^{\infty} d x_{2} \frac{L}{(\pi t)^{1 / 2}} \int_{x_{2}}^{\infty} d q e^{-q^{2} /(4 t)} \\
& =|S|-\frac{2 L t^{1 / 2}}{\pi^{1 / 2}}+O\left(t^{1 / 2} e^{-\delta^{2} /(4 t)}\right)
\end{aligned}
$$

This proves (2.5). The observation concluding the proof of Lemma 2.3 immediately implies Eq. 2.6.

Proof of Theorem 1.1 Similarly to the strategies of the proofs in [6-8], it remains to apply the model computations in Lemmas 2.2, 2.3 and 2.4, the sector computations from Theorem $1.2,[6]$ and [8] to the sets which partition $D$, and then apply Lemma 2.1 to the compensating terms.

We first choose $R$ and $\delta$ appropriately in the partition of $D$. Let $v$ be an arbitrary vertex of the polygonal boundary, and let $e_{v}$ denote the union of the two edges of $\partial D$ adjacent to $v$. We choose

$$
R=\frac{1}{2} \inf _{v \in \mathcal{V}} \inf \left\{d(v, y): y \in \partial D-e_{v}\right\} .
$$

This choice of $R$ guarantees that all circular sectors are non-overlapping. Moreover, the distance from any point in a circular sector with vertex $v$, radius $R$, and angle $\theta$ to $W_{\theta}-D$ is at least $R$. By Lemma 2.1 we have that the model computations for the sectors with angles in $\mathcal{A}, \mathcal{B}, \mathcal{C}$ give the appropriate contributions to $G_{D, \partial D_{-}}(t)$ in Eq. 1.15 up to an additive constant which is bounded in absolute value by $2|D| e^{-R^{2} /(4 t)}$.

Next we choose $\delta$ sufficiently small to ensure that the cusps are pairwise disjoint. We define $\varepsilon$ to be the smallest interior angle of the boundary $\partial D$ :

$$
\varepsilon=\min \{\mathcal{A} \cup \mathcal{B} \cup \mathcal{C}\} .
$$

It is straightforward to check that

$$
\delta=\frac{R}{2} \sin (\varepsilon / 2)
$$

satisfies the aforementioned condition.

The distance between the cusp and $H-D$ is larger than $\delta=\frac{R}{2} \sin (\varepsilon / 2)$ (if we consider the cusp corresponding to the sector with angle $\varepsilon$ ). By Lemma 2.1, we have that the model computations in Lemmas 2.2 and 2.3 give the appropriate contributions to $G_{D, \partial D_{-}}(t)$ up to an additive constant which is bounded in absolute value by $2|D| e^{-R^{2}(\sin (\varepsilon / 2))^{2} /(16 t)}$. This is because the terms of order $t^{3 / 2}$ and higher in Theorem 1.2, Lemmas 2.2 and 2.3 cancel out up to an exponentially small remainder.

Next we consider the contribution of the subset of $D$ which is within distance $\delta$ of $\partial D$, and which is not contained in any of the radial sectors and their corresponding cusps. This subset is a collection of disjoint rectangles supported either by a Dirichlet or an open edge 
respectively. Each such rectangle has at least distance $\delta$ to any of the other edges. We conclude that, by Lemmas 2.1 and 2.4, they give the various contributions to $G_{D, \partial D_{-}}(t)$ up to an additive constant which is bounded in absolute value by $2|D| e^{-R^{2}(\sin (\varepsilon / 2))^{2} /(16 t)}$.

The remaining subset of $D$ which is not contained in a sector, cusp or rectangle has distance $\delta$ to the boundary, and so contributes its measure up to an additive constant which is bounded in absolute value by $2|D| e^{-R^{2}(\sin (\varepsilon / 2))^{2} /(16 t)}$, by Lemma 2.1. All remainders above and in the proof of Theorem 1.2 are of the form $O\left(t^{\zeta} e^{-\eta / t}\right), \zeta \geq 0, \eta>0$. This gives the remainder in Eq. 1.15.

\section{Technical Preliminaries}

It has been noted (see p.43 in [8]) that there are three closed form expressions for the heat kernel of a wedge with opening angle $\gamma$, see [10, 15] and [18]. The authors of [8] were unable to extract the angle contribution $c(\gamma) t$ featuring in Eq. 1.13 from these expressions. In the case at hand, there is a fourth explicit formula for the heat kernel of a wedge with opening angle $2 \pi$ (see p.380 in [9]). However, we were unable to obtain a workable expression using that formula.

D. B. Ray managed to compute the angle contribution of the trace of the Dirichlet heat semigroup for a polygon using the Laplace transform of the heat kernel for a wedge, expressed as a Kontorovich Lebedev transform (see the footnote on p.44 of [16]). This strategy has been successfully employed in both [7] and [8]. We also employ it in this article.

Let $W_{\alpha}$ be the open infinite wedge as in Theorem 1.2, and let $p_{W_{\alpha}}\left(A_{1}, A_{2} ; t\right)$ denote the Dirichlet heat kernel for $W_{\alpha}$. Throughout we require $s>0$ and $t>0$. Let

$$
\hat{p}_{W_{\alpha}}\left(A_{1}, A_{2} ; s\right)=\int_{0}^{\infty} d t e^{-s t} p_{W_{\alpha}}\left(A_{1}, A_{2} ; t\right), s>0,
$$

be the associated Green's function (that is, the Laplace transform of $p_{W_{\alpha}}\left(A_{1}, A_{2} ; t\right)$ ), and let $A_{i}=\left(a_{i}, \alpha_{i}\right), i=1,2$ in polar coordinates. Then, following the footnote on p.44 in [16], and Appendix A of [17],

$$
\begin{aligned}
\hat{p}_{W_{\alpha}}\left(A_{1}, A_{2} ; s\right)= & \frac{1}{\pi^{2}} \int_{0}^{\infty} d \theta K_{i \theta}\left(\sqrt{s} a_{1}\right) K_{i \theta}\left(\sqrt{s} a_{2}\right) \\
& \times\left(\cosh \left(\left(\pi-\left|\alpha_{1}-\alpha_{2}\right|\right) \theta\right)-\frac{\sinh (\pi \theta)}{\sinh (\alpha \theta)} \cosh \left(\left(\alpha-\alpha_{1}-\alpha_{2}\right) \theta\right)\right. \\
& \left.+\frac{\sinh ((\pi-\alpha) \theta)}{\sinh (\alpha \theta)} \cosh \left(\left(\alpha_{1}-\alpha_{2}\right) \theta\right)\right)
\end{aligned}
$$

where $K_{i \theta}$ is the modified Bessel function, defined for example by formula 3.547.4 of [14],

$$
K_{i \theta}(\sqrt{s} a)=\int_{0}^{\infty} d w \cos (w \theta) e^{-\sqrt{s} a \cosh w}
$$


In the special case $\alpha=2 \pi$, Eq. 3.1 simplifies and we obtain

$$
\begin{aligned}
\hat{p}_{W_{2 \pi}}\left(A_{1}, A_{2} ; s\right)= & \frac{1}{\pi^{2}} \int_{0}^{\infty} d \theta K_{i \theta}\left(\sqrt{s} a_{1}\right) K_{i \theta}\left(\sqrt{s} a_{2}\right) \\
& \times\left(\cosh \left(\left(\pi-\left|\alpha_{1}-\alpha_{2}\right|\right) \theta\right)-\frac{\sinh (\pi \theta)}{\sinh (2 \pi \theta)}\left(\cosh \left(\left(2 \pi-\alpha_{1}-\alpha_{2}\right) \theta\right)\right.\right. \\
& \left.\left.+\cosh \left(\left(\alpha_{1}-\alpha_{2}\right) \theta\right)\right)\right) .
\end{aligned}
$$

In order to prove Theorem 1.2 in Section 4 below, we compute

$$
\int_{0}^{R} d a_{1} a_{1} \int_{0}^{\infty} d a_{2} a_{2} \int_{0}^{\alpha} d \alpha_{1} \int_{0}^{\alpha} d \alpha_{2} \hat{p}_{W_{2 \pi}}\left(A_{1}, A_{2} ; s\right),
$$

and then take the inverse Laplace transform. Throughout this paper we denote by $L^{-1}$ the inverse Laplace transform. That is, if $\hat{f}(s)=\int_{0}^{\infty} d t e^{-s t} f(t)$ then $L^{-1}\{\hat{f}\}(t)=f(t)$, at points of continuity of $f$.

The lack of a suitable Tauberian theorem prevents us from deducing the behaviour as $t \downarrow 0$ of $\int_{0}^{R} d r r \int_{0}^{\alpha} d \phi u_{W_{\alpha}}((r, \phi) ; t)$ from the behaviour as $s \uparrow \infty$ of the expression under Eq. 3.3. So after the computation of Eq. 3.3, the resulting $s$-dependent terms have to be inverted to the $t$-domain, including those terms which turn out to be exponentially small in $t$. For the reader's convenience, we list some relevant formulae for the computation of Eq. 3.3 above.

Formulae 6.561.16, 8.332.3 in [14] yield

$$
\int_{0}^{\infty} d a a K_{i \theta}(\sqrt{s} a)=\frac{\pi \theta}{2 s \sinh (\pi \theta / 2)} .
$$

Moreover formulae 6.794.2, 6.795.1, 4.114.2, 4.116.2 in [14] read

$$
\begin{gathered}
\int_{0}^{\infty} d \theta \cosh (\pi \theta / 2) K_{i \theta}(\sqrt{s} a)=\frac{\pi}{2}, a>0 \\
\int_{0}^{\infty} d \theta \cos (b \theta) K_{i \theta}(a)=\frac{\pi}{2} e^{-a \cosh b}, a>0,|\Im b| \leq \frac{\pi}{2} \\
\int_{0}^{\infty} d \theta \frac{\cos (a \theta)}{\theta} \frac{\sinh (\beta \theta)}{\cosh (\gamma \theta)}=\frac{1}{2} \log \left(\frac{\cosh (a \pi /(2 \gamma))+\sin (\beta \pi /(2 \gamma))}{\cosh (a \pi /(2 \gamma))-\sin (\beta \pi /(2 \gamma))}\right),|\Re \beta|<\Re \gamma . \\
\int_{0}^{\infty} \frac{d \theta}{\theta} \cos (a \theta) \tanh (\beta \theta)=\log \operatorname{coth}(a \pi /(4 \beta))
\end{gathered}
$$

where $\Re \beta$, respectively $\Im \beta$, denotes the real, respectively imaginary, part of $\beta$.

Finally, formula 5.6.3 in [11] reads

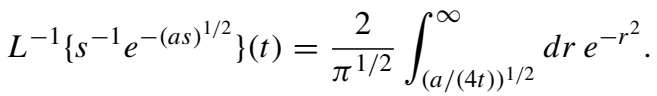

\section{Proof of Theorem 1.2}

\section{Proof of Theorem 1.2}

As described in Section 3, we compute

$$
\int_{0}^{R} d a_{1} a_{1} \int_{0}^{\infty} d a_{2} a_{2} \int_{0}^{\alpha} d \alpha_{1} \int_{0}^{\alpha} d \alpha_{2} \hat{p}_{W_{2 \pi}}\left(A_{1}, A_{2} ; s\right),
$$


where

$$
\begin{aligned}
\hat{p}_{W_{2 \pi}}\left(A_{1}, A_{2} ; s\right)= & \frac{1}{\pi^{2}} \int_{0}^{\infty} d \theta K_{i \theta}\left(\sqrt{s} a_{1}\right) K_{i \theta}\left(\sqrt{s} a_{2}\right) \\
& \times\left(\cosh \left(\left(\pi-\left|\alpha_{1}-\alpha_{2}\right|\right) \theta\right)-\frac{\sinh (\pi \theta)}{\sinh (2 \pi \theta)}\left(\cosh \left(\left(2 \pi-\alpha_{1}-\alpha_{2}\right) \theta\right)\right.\right. \\
& \left.\left.+\cosh \left(\left(\alpha_{1}-\alpha_{2}\right) \theta\right)\right)\right),
\end{aligned}
$$

and then take the inverse Laplace transform. A straightforward computation shows

$$
\begin{aligned}
& \int_{0}^{\alpha} d \alpha_{1} \int_{0}^{\alpha} d \alpha_{2}\left(\cosh \left(\left(\pi-\left|\alpha_{1}-\alpha_{2}\right|\right) \theta\right)-\frac{\sinh (\pi \theta)}{\sinh (2 \pi \theta)}\left(\cosh \left(\left(2 \pi-\alpha_{1}-\alpha_{2}\right) \theta\right)\right.\right. \\
& \left.\left.+\cosh \left(\left(\alpha_{1}-\alpha_{2}\right) \theta\right)\right)\right) \\
= & \frac{2 \alpha}{\theta} \sinh (\pi \theta)+\frac{1}{2 \theta^{2} \cosh (\pi \theta)}(3-3 \cosh (2 \pi \theta)) \\
& +\frac{1}{2 \theta^{2} \cosh (\pi \theta)}(4 \cosh ((2 \pi-\alpha) \theta)-\cosh ((2 \pi-2 \alpha) \theta)-3) \\
:= & C_{1}+C_{2}+C_{3},
\end{aligned}
$$

with obvious notation.

We obtain by definition of $C_{1}$, Fubini's theorem, Eqs. 3.4 and 3.5,

$$
\begin{aligned}
& \int_{0}^{R} d a_{1} a_{1} \int_{0}^{\infty} d a_{2} a_{2} \frac{1}{\pi^{2}} \int_{0}^{\infty} d \theta K_{i \theta}\left(\sqrt{s} a_{1}\right) K_{i \theta}\left(\sqrt{s} a_{2}\right) C_{1} \\
= & \frac{2 \alpha}{\pi s} \int_{0}^{R} d a_{1} a_{1} \int_{0}^{\infty} d \theta K_{i \theta}\left(\sqrt{s} a_{1}\right) \cosh (\pi \theta / 2) \\
= & \frac{\alpha R^{2}}{2 s} .
\end{aligned}
$$

So $L^{-1}\left\{(2 s)^{-1} \alpha R^{2}\right\}(t)=2^{-1} \alpha R^{2}$, which is the first term in right-hand side of Eq. 1.17.

Furthermore, by Fubini's theorem, Eq. 3.4, and the definition of $C_{2}$ in Eq. 4.1, we find

$$
\begin{aligned}
& \int_{0}^{R} d a_{1} a_{1} \int_{0}^{\infty} d a_{2} a_{2} \frac{1}{\pi^{2}} \int_{0}^{\infty} d \theta K_{i \theta}\left(\sqrt{s} a_{1}\right) K_{i \theta}\left(\sqrt{s} a_{2}\right) C_{2} \\
= & -\frac{3}{\pi^{2}} \int_{0}^{R} d a_{1} a_{1} \int_{0}^{\infty} d a_{2} a_{2} \int_{0}^{\infty} \frac{d \theta}{\theta^{2}} K_{i \theta}\left(\sqrt{s} a_{1}\right) K_{i \theta}\left(\sqrt{s} a_{2}\right) \tanh (\pi \theta) \sinh (\pi \theta) \\
= & -\frac{3}{\pi} \int_{0}^{R} d a_{1} a_{1} \int_{0}^{\infty} \frac{d \theta}{\theta s} K_{i \theta}\left(\sqrt{s} a_{1}\right) \tanh (\pi \theta) \cosh (\pi \theta / 2) \\
= & -\frac{3}{\pi} \int_{0}^{R} d a_{1} a_{1} \int_{0}^{\infty} \frac{d \theta}{\theta s} K_{i \theta}\left(\sqrt{s} a_{1}\right)\left(\sinh (\pi \theta / 2)+\frac{\sinh (\pi \theta / 2)}{\cosh (\pi \theta)}\right) .
\end{aligned}
$$

See also (2.9) in [8]. By Eq. 3.6, and Fubini's theorem (see (2.10) in [8]),

$$
\begin{aligned}
-\frac{3}{\pi s} \int_{0}^{\infty} \frac{d \theta}{\theta} K_{i \theta}\left(\sqrt{s} a_{1}\right) \sinh (\pi \theta / 2) & =-\frac{3}{\pi s} \int_{0}^{\pi / 2} d \eta \int_{0}^{\infty} d \theta \cosh (\eta \theta) K_{i \theta}\left(\sqrt{s} a_{1}\right) \\
& =-\frac{3}{2 s} \int_{0}^{\pi / 2} d \eta e^{-a_{1} \sqrt{s} \cos \eta}
\end{aligned}
$$


By Eq. 4.3, we obtain for the first term in the right-hand side of Eq. 4.2

$$
\begin{gathered}
-\frac{3}{\pi} \int_{0}^{R} d a_{1} a_{1} \int_{0}^{\infty} \frac{d \theta}{\theta s} K_{i \theta}\left(\sqrt{s} a_{1}\right) \sinh (\pi \theta / 2) \\
=-\frac{3}{2 s} \int_{0}^{R} d a a \int_{0}^{\pi / 2} d \eta e^{-a \sqrt{s} \cos \eta} .
\end{gathered}
$$

From the calculation in (2.14) of [8], we find that the inverse Laplace transform of the right-hand side of Eq. 4.4 is given by

$$
-\frac{3 R t^{1 / 2}}{\pi^{1 / 2}}+\frac{3 R t^{1 / 2}}{\pi^{1 / 2}} \int_{1}^{\infty} \frac{d v}{v^{2}} \int_{0}^{1} \frac{\zeta d \zeta}{\left(1-\zeta^{2}\right)^{1 / 2}} e^{-R^{2} \zeta^{2} v^{2} /(4 t)} .
$$

For the second term in the right-hand side of Eq. 4.2, by Fubini's theorem and Eq. 3.4, we have

$$
\begin{aligned}
- & \frac{3}{\pi} \int_{0}^{R} d a_{1} a_{1} \int_{0}^{\infty} \frac{d \theta}{\theta s} K_{i \theta}\left(\sqrt{s} a_{1}\right) \frac{\sinh (\pi \theta / 2)}{\cosh (\pi \theta)} \\
& =-\frac{3}{2 s^{2}} \int_{0}^{\infty} \frac{d \theta}{\cosh (\pi \theta)}+\frac{3}{\pi} \int_{R}^{\infty} d a_{1} a_{1} \int_{0}^{\infty} \frac{d \theta}{\theta s} K_{i \theta}\left(\sqrt{s} a_{1}\right) \frac{\sinh (\pi \theta / 2)}{\cosh (\pi \theta)} \\
& =-\frac{3}{4 s^{2}}+\frac{3}{\pi} \int_{R}^{\infty} d a_{1} a_{1} \int_{0}^{\infty} \frac{d \theta}{\theta s} K_{i \theta}\left(\sqrt{s} a_{1}\right) \frac{\sinh (\pi \theta / 2)}{\cosh (\pi \theta)} .
\end{aligned}
$$

Taking the inverse Laplace transform of the first term in the right-hand side of Eq. 4.5 yields $-\frac{3}{4} t$, which accounts for the $-\frac{3}{4}$ term in Eq. 1.16.

By Fubini's theorem and Eq. 3.2, we obtain

$$
\begin{aligned}
& \frac{3}{\pi} \int_{R}^{\infty} d a a \int_{0}^{\infty} \frac{d \theta}{\theta s} K_{i \theta}(\sqrt{s} a) \frac{\sinh (\pi \theta / 2)}{\cosh (\pi \theta)} \\
= & \frac{3}{\pi} \int_{R}^{\infty} d a a \int_{0}^{\infty} d w e^{-\sqrt{s} a \cosh w} \int_{0}^{\infty} \frac{d \theta}{\theta s} \cos (w \theta) \frac{\sinh (\pi \theta / 2)}{\cosh (\pi \theta)} .
\end{aligned}
$$

By Eq. 3.9,

$$
L^{-1}\left\{s^{-1} e^{-\sqrt{s} a \cosh w}\right\}(t)=\frac{2}{\sqrt{\pi}} \int_{(a \cosh w) /(4 t)^{1 / 2}}^{\infty} d r e^{-r^{2}} .
$$

Hence the inverse Laplace transform of the second term in the right-hand side of Eq. 4.5 is bounded in absolute value by

$$
\begin{aligned}
& \frac{3}{\pi}\left|\int_{R}^{\infty} d a a \int_{0}^{\infty} d w \operatorname{Erfc}\left((a \cosh w) /(4 t)^{1 / 2}\right) \int_{0}^{\infty} \frac{d \theta}{\theta} \cos (w \theta) \frac{\sinh (\pi \theta / 2)}{\cosh (\pi \theta)}\right| \\
\leq & \frac{3}{\pi} \int_{R}^{\infty} d a a \int_{0}^{\infty} d w \operatorname{Erfc}\left((a \cosh w) /(4 t)^{1 / 2}\right) \int_{0}^{\infty} \frac{d \theta}{\theta} \frac{\sinh (\pi \theta / 2)}{\cosh (\pi \theta)} \\
= & \frac{3 \log (1+\sqrt{2})}{\pi} \int_{0}^{\infty} d w \int_{R}^{\infty} d a a \operatorname{Erfc}\left((a \cosh w) /(4 t)^{1 / 2}\right),
\end{aligned}
$$

where we have used Eq. 3.7. Since for $z \geq 0$

$$
\operatorname{Erfc}(z):=\frac{2}{\sqrt{\pi}} \int_{z}^{\infty} d r e^{-r^{2}}=\frac{2}{\sqrt{\pi}} \int_{0}^{\infty} d r e^{-(z+r)^{2}} \leq \frac{2}{\sqrt{\pi}} \int_{0}^{\infty} d r e^{-z^{2}-r^{2}}=e^{-z^{2}},
$$


we obtain that the right-hand side of Eq. 4.7 is bounded from above by

$$
\begin{aligned}
\frac{3 \log (1+\sqrt{2})}{\pi} & \int_{0}^{\infty} d w \int_{R}^{\infty} d a a e^{-(a \cosh w)^{2} /(4 t)} \\
& =\frac{6 t \log (1+\sqrt{2})}{\pi} \int_{0}^{\infty} \frac{d w}{(\cosh w)^{2}} e^{-(R \cosh w)^{2} /(4 t)} \\
& =O\left(t e^{-R^{2} /(4 t)}\right)
\end{aligned}
$$

In order to compute $C_{3}$, we extend the integral with respect to $a_{1}$ to the interval $[0, \infty)$, and obtain, via Fubini's theorem and Eq. 3.4,

$$
\begin{aligned}
& \int_{0}^{\infty} d a_{1} a_{1} \int_{0}^{\infty} d a_{2} a_{2} \frac{1}{\pi^{2}} \int_{0}^{\infty} d \theta K_{i \theta}\left(\sqrt{s} a_{1}\right) K_{i \theta}\left(\sqrt{s} a_{2}\right) C_{3} \\
= & \int_{0}^{\infty} d a_{1} a_{1} \int_{0}^{\infty} d a_{2} a_{2} \frac{1}{\pi^{2}} \int_{0}^{\infty} d \theta K_{i \theta}\left(\sqrt{s} a_{1}\right) K_{i \theta}\left(\sqrt{s} a_{2}\right) \\
& \times \frac{1}{2 \theta^{2} \cosh (\pi \theta)}(4 \cosh ((2 \pi-\alpha) \theta)-\cosh ((2 \pi-2 \alpha) \theta)-3) \\
= & \frac{1}{8 s^{2}} \int_{0}^{\infty} d \theta \frac{4 \cosh ((2 \pi-\alpha) \theta)-\cosh ((2 \pi-2 \alpha) \theta)-3}{\cosh (\pi \theta)(\sinh (\pi \theta / 2))^{2}} \\
= & \frac{1}{4 s^{2}} \int_{0}^{\infty} d \theta \frac{4\left(\sinh \left(\left(\pi-\frac{\alpha}{2}\right) \theta\right)\right)^{2}-(\sinh ((\pi-\alpha) \theta))^{2}}{(\sinh (\pi \theta / 2))^{2} \cosh (\pi \theta)} .
\end{aligned}
$$

Inverting the Laplace transform yields a contribution $\left(\frac{3}{4}+a(\alpha)\right) t$, where $a(\alpha)$ is as defined in Eq. 1.16. This, together with the statement below Eq. 4.5 gives the contribution $a(\alpha) t$ in Eq. 1.17 .

It remains to bound the inverse Laplace transform of

$$
\begin{aligned}
& \int_{R}^{\infty} d a_{1} a_{1} \int_{0}^{\infty} d a_{2} a_{2} \frac{1}{\pi^{2}} \int_{0}^{\infty} d \theta K_{i \theta}\left(\sqrt{s} a_{1}\right) K_{i \theta}\left(\sqrt{s} a_{2}\right) \\
& \times \frac{1}{2 \theta^{2} \cosh (\pi \theta)}(4 \cosh ((2 \pi-\alpha) \theta)-\cosh ((2 \pi-2 \alpha) \theta)-3) \\
= & \frac{1}{s} \int_{R}^{\infty} d a a \int_{0}^{\infty} d \theta K_{i \theta}(\sqrt{s} a) \frac{4 \cosh ((2 \pi-\alpha) \theta)-\cosh ((2 \pi-2 \alpha) \theta)-3}{4 \pi \theta \sinh (\pi \theta / 2) \cosh (\pi \theta)} .
\end{aligned}
$$

We first consider the case $\pi / 2<\alpha<7 \pi / 4$, and we proceed as above. We use Eq. 3.2, and invert the Laplace transform of $s^{-1} e^{-\sqrt{s} a \cosh w}$ as in Eq. 4.6. This gives that the inverse Laplace transform of Eq. 4.10 equals

$$
\begin{aligned}
\int_{R}^{\infty} d a a \int_{0}^{\infty} d w \operatorname{Erfc}\left((a \cosh w) /(4 t)^{1 / 2}\right) \int_{0}^{\infty} d \theta(\cos (w \theta)) \\
\times \frac{4 \cosh ((2 \pi-\alpha) \theta)-\cosh ((2 \pi-2 \alpha) \theta)-3}{4 \pi \theta \sinh (\pi \theta / 2) \cosh (\pi \theta)} .
\end{aligned}
$$

Using $|\cos (w \theta)| \leq 1$, we find that the absolute value of the expression under Eq. 4.11 is bounded from above by

$$
\begin{aligned}
\int_{R}^{\infty} \operatorname{daa} \int_{0}^{\infty} d w \operatorname{Erfc}(( & \left.\cosh w) /(4 t)^{1 / 2}\right) \\
& \times \int_{0}^{\infty} d \theta \frac{4 \cosh ((2 \pi-\alpha) \theta)-\cosh ((2 \pi-2 \alpha) \theta)-3}{4 \pi \theta \sinh (\pi \theta / 2) \cosh (\pi \theta)} \\
= & O\left(t e^{-R^{2} /(4 t)}\right)
\end{aligned}
$$


where, as before, we have used Eq. 4.8, and argued similarly to Eq. 4.9. We note that the integrals with respect to $\theta$ in Eqs. 4.11 and 4.12 converge for $\pi / 2<\alpha<7 \pi / 4$.

We next consider the case $7 \pi / 4<\alpha<2 \pi$. We write the right-hand side of Eq. 4.10 as the sum of two terms, say $D_{1}(s)+D_{2}(s)$, where

$$
D_{1}(s)=\frac{1}{s} \int_{R}^{\infty} d a a \int_{0}^{\infty} d \theta K_{i \theta}(\sqrt{s} a) \frac{\cosh ((2 \pi-\alpha) \theta)-1}{\pi \theta \sinh (\pi \theta / 2) \cosh (\pi \theta)},
$$

and

$$
D_{2}(s)=\frac{1}{s} \int_{R}^{\infty} d a a \int_{0}^{\infty} d \theta K_{i \theta}(\sqrt{s} a) \frac{1-\cosh ((2 \pi-2 \alpha) \theta)}{4 \pi \theta \sinh (\pi \theta / 2) \cosh (\pi \theta)} .
$$

Using Eqs. 3.2, 4.6 gives

$$
\begin{aligned}
\left|L^{-1}\left\{D_{1}\right\}(t)\right|= & \mid \int_{R}^{\infty} d a a \int_{0}^{\infty} d w \operatorname{Erfc}\left((a \cosh w) /(4 t)^{1 / 2}\right) \\
& \times \int_{0}^{\infty} d \theta \cos (w \theta) \frac{\cosh ((2 \pi-\alpha) \theta)-1}{\pi \theta \sinh (\pi \theta / 2) \cosh (\pi \theta)} \mid \\
\leq & \int_{R}^{\infty} d a a \int_{0}^{\infty} d w \operatorname{Erfc}\left((a \cosh w) /(4 t)^{1 / 2}\right) \\
& \times \int_{0}^{\infty} d \theta \frac{\cosh ((2 \pi-\alpha) \theta)-1}{\pi \theta \sinh (\pi \theta / 2) \cosh (\pi \theta)} \\
= & O\left(t e^{-R^{2} /(4 t)}\right),
\end{aligned}
$$

where we have used Eq. 4.8, and argued similarly to Eq. 4.9. The integral with respect to $\theta$ in Eq. 4.15 converges for $\alpha \in(\pi / 2,2 \pi) \supset(7 \pi / 4,2 \pi)$. To invert $D_{2}(s)$ we rewrite the integrand as follows. For $\epsilon \in \mathbb{R}$,

$$
\begin{aligned}
\frac{1-\cosh ((2 \pi-2 \alpha) \theta)}{4 \pi \theta \sinh (\pi \theta / 2) \cosh (\pi \theta)} \\
=\frac{4 \cosh (\pi \theta / 2)-2 \cosh \left(\left(2 \alpha-\frac{3 \pi}{2}\right) \theta\right)-2 \cosh \left(\left(2 \alpha-\frac{5 \pi}{2}\right) \theta\right)}{4 \pi \theta \sinh (2 \pi \theta)} \\
=\frac{4 \cosh (\pi \theta / 2)-2 \cosh \left(\left(2 \alpha-\frac{3 \pi}{2}\right) \theta\right)-2 \cosh \left(\left(2 \alpha-\frac{5 \pi}{2}\right) \theta\right)}{4 \pi \theta \sinh (2 \pi \theta)} \\
\quad+\frac{2 \cosh ((2 \pi+\epsilon) \theta)-2 \cosh ((2 \pi-\epsilon) \theta)}{4 \pi \theta \sinh (2 \pi \theta)}-\frac{1}{\pi \theta} \sinh (\epsilon \theta) .
\end{aligned}
$$

We choose $2 \alpha-\frac{3 \pi}{2}=2 \pi+\epsilon$. This gives that $\epsilon=2 \alpha-\frac{7 \pi}{2}$, and

$$
\begin{aligned}
& \frac{1-\cosh ((2 \pi-2 \alpha) \theta)}{4 \pi \theta \sinh (\pi \theta / 2) \cosh (\pi \theta)} \\
& =\frac{2 \cosh (\pi \theta / 2)-\cosh \left(\left(2 \alpha-\frac{5 \pi}{2}\right) \theta\right)-\cosh \left(\left(\frac{11 \pi}{2}-2 \alpha\right) \theta\right)}{2 \pi \theta \sinh (2 \pi \theta)} \\
& \quad-\frac{1}{\pi \theta} \sinh ((4 \alpha-7 \pi) \theta / 2) .
\end{aligned}
$$

The first term in the right-hand side of Eq. 4.16 is integrable, and, analogously to the above, we proceed with Eqs. 3.2, 4.6 and 4.8. This gives a remainder $O\left(t e^{-R^{2} /(4 t)}\right)$. 
It remains to invert the contribution coming from the second term in the right-hand side of Eq. 4.16. We recall (2.18) in [8]. That is, for $-\frac{\pi}{2}<\beta<\frac{\pi}{2}$, by Fubini's theorem, Eqs. 3.6 and 3.9, we have

$$
\begin{aligned}
L^{-1}\left\{\int_{R}^{\infty} d a a \int_{0}^{\infty}\right. & \left.\frac{d \theta}{\theta s} K_{i \theta}(\sqrt{s} a) \sinh (\beta \theta)\right\}(t) \\
& =L^{-1}\left\{\int_{R}^{\infty} d a \frac{a}{s} \int_{0}^{\beta} d \eta \int_{0}^{\infty} d \theta \cosh (\eta \theta) K_{i \theta}(\sqrt{s} a)\right\}(t) \\
& =L^{-1}\left\{\frac{\pi}{2 s} \int_{R}^{\infty} d a a \int_{0}^{\beta} d \eta e^{-a \sqrt{s} \cos \eta}\right\}(t) \\
& =\frac{\pi}{2} \int_{R}^{\infty} d a a \int_{0}^{\beta} d \eta \operatorname{Erfc}\left(\frac{a \cos \eta}{(4 t)^{1 / 2}}\right) \\
& \leq \frac{\pi \beta}{2} \int_{R}^{\infty} d a a \operatorname{Erfc}\left(\frac{a \cos \beta}{(4 t)^{1 / 2}}\right) \\
& =O\left(t e^{-R^{2}(\cos \beta)^{2} /(4 t)}\right)
\end{aligned}
$$

where we have used once more Eq. 4.8.

For $7 \pi / 4<\alpha<2 \pi$ we have that $2 \alpha-\frac{7 \pi}{2} \in(0, \pi / 2)$. Hence the second term in the right-hand side of Eq. 4.16 gives a contribution $O\left(t e^{-R^{2}(\sin (2 \alpha))^{2} /(4 t)}\right)$.

For $\alpha=\frac{7 \pi}{4}$, we have

$$
\int_{0}^{\infty} d \theta\left|\frac{\cosh ((2 \pi-\alpha) \theta)-1}{\pi \theta \sinh (\pi \theta / 2) \cosh (\pi \theta)}\right|<\infty
$$

Hence the inverse Laplace transform of $D_{1}$ is $O\left(t e^{-R^{2} /(4 t)}\right)$. For $\alpha=7 \pi / 4$ we rewrite Eq. 4.14 as

$$
\begin{aligned}
D_{2} & =\frac{1}{s} \int_{R}^{\infty} d a a \int_{0}^{\infty} d \theta K_{i \theta}(\sqrt{s} a) \frac{\cosh (\pi \theta / 2)-\frac{1}{2} \cosh (\pi \theta)-\frac{1}{2} \cosh (2 \pi \theta)}{\pi \theta \sinh (2 \pi \theta)} \\
& =\frac{1}{s} \int_{R}^{\infty} d a a \int_{0}^{\infty} d \theta K_{i \theta}(\sqrt{s} a)\left\{\frac{\cosh (\pi \theta / 2)-\frac{1}{2} \cosh (\pi \theta)-\frac{1}{2}}{\pi \theta \sinh (2 \pi \theta)}-\frac{\tanh (\pi \theta)}{2 \pi \theta}\right\} .
\end{aligned}
$$

Since

$$
\int_{0}^{\infty} d \theta\left|\frac{\cosh (\pi \theta / 2)-\frac{1}{2} \cosh (\pi \theta)-\frac{1}{2}}{\pi \theta \sinh (2 \pi \theta)}\right|<\infty,
$$

we have that this part also gives a contribution $O\left(t e^{-R^{2} /(4 t)}\right)$. By Eqs. 3.8 and 3.9 (see (2.20) in [8]), we have for $\beta>0$,

$$
\begin{aligned}
& L^{-1}\left\{\frac{1}{\pi s} \int_{R}^{\infty} d a a \int_{0}^{\infty} \frac{d \theta}{\theta} \tanh (\beta \theta) K_{i \theta}(\sqrt{s} a)\right\}(t) \\
= & L^{-1}\left\{\frac{1}{\pi s} \int_{R}^{\infty} d a a \int_{0}^{\infty} d w e^{-a \sqrt{s} \cosh w} \int_{0}^{\infty} \frac{d \theta}{\theta} \tanh (\beta \theta) \cos (w \theta)\right\}(t) \\
= & L^{-1}\left\{\frac{1}{\pi s} \int_{R}^{\infty} d a a \int_{0}^{\infty} d w e^{-a \sqrt{s} \cosh w} \log (\operatorname{coth}(\pi w /(4 \beta)))\right\}(t) \\
= & \frac{1}{\pi} \int_{R}^{\infty} d a a \int_{0}^{\infty} d w \operatorname{Erfc}\left(\frac{a \cosh w}{(4 t)^{1 / 2}}\right) \log (\operatorname{coth}(\pi w /(4 \beta))) .
\end{aligned}
$$


By Eq. 4.8 we obtain that Eq. 4.18 is bounded from above by

$$
2 \pi^{-1} t e^{-R^{2} /(4 t)} \int_{0}^{\infty} d w \frac{\log (\operatorname{coth}(\pi w /(4 \beta)))}{(\cosh w)^{2}}=O\left(t e^{-R^{2} /(4 t)}\right) .
$$

In particular, for $\beta=\pi$, the term $-\frac{\tanh (\pi \theta)}{2 \pi \theta}$ contributes a remainder $O\left(t e^{-R^{2} /(4 t)}\right)$.

For $\alpha=\pi / 2$, we have

$$
\int_{0}^{\infty}\left|\frac{1-\cosh ((2 \pi-\alpha) \theta)}{4 \pi \theta \sinh (\pi \theta / 2) \cosh (\pi \theta)}\right|<\infty
$$

Hence the inverse Laplace transform of $D_{2}$ is, for $\alpha=\pi / 2, O\left(t e^{-R^{2} /(4 t)}\right)$. On the other hand, for $\alpha=\pi / 2$ the integrand in Eq. 4.13 equals the integrand of Eq. 4.14 for $\alpha=7 \pi / 4$ up to a factor of $-\frac{1}{4}$. Hence the inverse Laplace transform of $D_{1}$ is also $O\left(t e^{-R^{2} /(4 t)}\right)$.

For $\pi / 4<\alpha<\pi / 2$, we have

$$
\int_{0}^{\infty}\left|\frac{1-\cosh ((2 \pi-2 \alpha) \theta)}{4 \pi \theta \sinh (\pi \theta / 2) \cosh (\pi \theta)}\right|<\infty
$$

Hence the inverse Laplace transform of $D_{2}$ is, for $\pi / 4<\alpha<\pi / 2, O\left(t e^{-R^{2} /(4 t)}\right)$. Similarly to the above, we rewrite the hyperbolic part of the integrand in Eq. 4.13 as follows:

$$
\begin{aligned}
\frac{\cosh ((2 \pi-\alpha) \theta)-1}{\pi \theta \sinh (\pi \theta / 2) \cosh (\pi \theta)} \\
=\frac{2 \cosh \left(\left(\frac{5 \pi}{2}-\alpha\right) \theta\right)+2 \cosh \left(\left(\frac{3 \pi}{2}-\alpha\right) \theta\right)-4 \cosh (\pi \theta / 2)}{\pi \theta \sinh (2 \pi \theta)} \\
=\frac{2 \cosh \left(\left(\frac{5 \pi}{2}-\alpha\right) \theta\right)+2 \cosh \left(\left(\frac{3 \pi}{2}-\alpha\right) \theta\right)-4 \cosh (\pi \theta / 2)}{\pi \theta \sinh (2 \pi \theta)} \\
\quad+\frac{-2 \cosh ((2 \pi+\epsilon) \theta)+2 \cosh ((2 \pi-\epsilon) \theta)}{\pi \theta \sinh (2 \pi \theta)} \\
\quad+\frac{4 \sinh (\epsilon \theta)}{\pi \theta} .
\end{aligned}
$$

We subsequently choose $\epsilon=\frac{\pi}{2}-\alpha$. With this choice of $\epsilon$, the absolute value of the first term in the right-hand side of Eq. 4.19 is integrable with respect to $\theta$ on $\mathbb{R}^{+}$. Hence this term contributes $O\left(t e^{-R^{2} /(4 t)}\right)$ to the inverse Laplace transform of the corresponding integral in Eq. 4.13. Moreover, since $\epsilon \in(0, \pi / 2)$ for this case, we have by Eq. 4.17 that this term contributes $O\left(t e^{-R^{2} \sin ^{2}(\alpha) /(4 t)}\right)$ to the inverse Laplace transform of the corresponding integral in Eq. 4.13.

We next consider the case $\alpha=\pi / 4$. Then $D_{2}$ for $\pi / 4$ equals $D_{2}$ for $7 \pi / 4$, we immediately conclude that this term is $O\left(t e^{-R^{2} /(4 t)}\right)$. We rewrite the hyperbolic part of the integrand as follows: 


$$
\begin{aligned}
\frac{\cosh (7 \pi \theta / 4)-1}{\pi \theta \sinh (\pi \theta / 2) \cosh (\pi \theta)} & \\
= & \frac{2 \cosh (9 \pi \theta / 4)+2 \cosh (5 \pi \theta / 4)-2 \cosh ((2 \pi+\epsilon) \theta)}{\pi \theta \sinh (2 \pi \theta)} \\
& +\frac{2 \cosh ((2 \pi-\epsilon) \theta)-4 \cosh (\pi \theta / 2)}{\pi \theta \sinh (2 \pi \theta)} \\
& +\frac{4 \sinh (\epsilon \theta)}{\pi \theta} .
\end{aligned}
$$

We subsequently choose $\epsilon=\frac{\pi}{4}$ and observe that the absolute value of the first term in the right-hand side of Eq. 4.20 is integrable. This then yields that the corresponding Laplace transform is $O\left(t e^{-R^{2} /(4 t)}\right)$. The second term has been inverted in Eq. 4.17. Choosing $\beta=$ $\pi / 4$ gives a remainder $O\left(t e^{-R^{2} /(8 t)}\right)$.

We finally consider the case $0<\alpha<\pi / 4$. The contribution from $D_{1}$ to the inverse Laplace transform can be estimated by Eq. 4.19, and the lines below, since $\epsilon \in(0, \pi / 2)$ for this case too. Hence we obtain a remainder $O\left(t e^{-R^{2}(\sin \alpha)^{2} /(4 t)}\right)$. The contribution from $D_{2}$ to the inverse Laplace transform follows by a minor modification of Eq. 4.19. We have

$$
\begin{aligned}
\frac{1-\cosh ((2 \pi-2 \alpha) \theta)}{4 \pi \theta \sinh (\pi \theta / 2) \cosh (\pi \theta)} \\
=\frac{\cosh (\pi \theta / 2)-\frac{1}{2} \cosh \left(\left(\frac{5 \pi}{2}-2 \alpha\right) \theta\right)-\frac{1}{2} \cosh \left(\left(\frac{3 \pi}{2}-2 \alpha\right) \theta\right)}{\pi \theta \sinh (2 \pi \theta)} \\
\quad+\frac{\frac{1}{2} \cosh ((2 \pi+\epsilon) \theta)-\frac{1}{2} \cosh ((2 \pi-\epsilon) \theta)}{\pi \theta \sinh (2 \pi \theta)} \\
\quad-\frac{\sinh (\epsilon \theta)}{\pi \theta} .
\end{aligned}
$$

We choose $\epsilon=\frac{\pi}{2}-2 \alpha \in(0, \pi / 2)$, and obtain the remainder $O\left(t e^{-R^{2} \sin ^{2}(2 \alpha) /(4 t)}\right)$ from the corresponding integral in Eq. 4.14 by Eq. 4.17. The first term in the right-hand side of Eq. 4.21 gives $O\left(t e^{-R^{2} /(4 t)}\right)$.

Acknowledgments MvdB was supported by a Leverhulme Trust Emeritus Fellowship EM-2018-011-9. MvdB acknowledges hospitality by the Max Planck Institute for Mathematics, Bonn, and the Mathematical Institute, University of Neuchâtel. KG acknowledges support from the Max Planck Institute for Mathematics, Bonn, from October 2017 to July 2018. The authors wish to thank the referee for helpful suggestions.

Open Access This article is distributed under the terms of the Creative Commons Attribution 4.0 International License (http://creativecommons.org/licenses/by/4.0/), which permits unrestricted use, distribution, and reproduction in any medium, provided you give appropriate credit to the original author(s) and the source, provide a link to the Creative Commons license, and indicate if changes were made.

\section{References}

1. van den Berg, M.: Heat flow and perimeter in $\mathbb{R}^{m}$. Potential Anal. 39, 369-387 (2013)

2. van den Berg, M., Davies, E.B.: Heat flow out of regions in $\mathbb{R}^{m}$. Math. Z. 202, 463-482 (1989)

3. van den Berg, M., Gilkey, P.B.: Heat flow out of a compact manifold. J. Geom. Anal. 25, 1576-1601 (2015) 
4. van den Berg, M., Gilkey, P., Kirsten, K., Kozlov, V.A.: Heat content asymptotics for Riemannian manifolds with Zaremba boundary conditions. Potential Anal. 26, 225-254 (2007)

5. van den Berg, M., Gittins, K.: Uniform bounds for the heat content of open sets in Euclidean space. Differential Geometry and its Applications 40, 67-85 (2015)

6. van den Berg, M., Gittins, K.: On the heat content of a polygon. J. Geom. Anal. 26, 2231-2264 (2016)

7. van den Berg, M., Srisatkunarajah, S.: Heat equation for a region in $\mathbb{R}^{2}$ with a polygonal boundary. J. Lond. Math. Soc. (2) 37, 119-127 (1988)

8. van den Berg, M., Srisatkunarajah, S.: Heat flow and Brownian motion for a region in $\mathbb{R}^{2}$ with a polygonal boundary. Probab. Theory Relat. Fields 86, 41-52 (1990)

9. Carslaw, H.S., Jaeger, J.C.: Conduction of Heat in Solids. Clarendon Press, Oxford (2000)

10. Cooke, J.C.: Note on a heat conduction problem. Amer. Math. Monthly 62, 331-334 (1955)

11. Erdélyi, A.: Tables of Integral Transforms I. McGraw-Hill, New York (1954)

12. Grigor'yan, A.: Heat kernel and analysis on manifolds AMS/IP studies in advanced mathematics, 47. American Mathematical Society, Providence, RI; International Press, Boston, MA (2009)

13. Gilkey, P.: Asymptotic Formulae in Spectral Geometry, Stud. Adv. Math. Chapman \& Hall/CRC, Boca Raton (2004)

14. Gradshteyn, I.S., Ryzhik, I.M.: Table of Integrals, Series, and Products. Elsevier/Academic Press, Amsterdam (2015)

15. Kac, M.: Can one hear the shape of a drum? Amer. Math. Monthly 73, 1-23 (1966)

16. McKean, H.P., Singer, I.M.: Curvature and the eigenvalues of the Laplacian. J. Differ. Geom. 1, 43-69 (1967)

17. Nursultanov, M., Rowlett, J., Sher, D.A.: The heat kernel on curvilinear polygonal domains in surfaces. arXiv:1905.00259 [math.AP] (2019)

18. Spitzer, F.: Some theorems concerning 2-dimensional Brownian motion. Trans. Amer. Math. Soc. 87, 187-197 (1958)

Publisher's Note Springer Nature remains neutral with regard to jurisdictional claims in published maps and institutional affiliations. 